\title{
OSCILLATORY PHENOMENA ASSOCIATED TO SEMILINEAR WAVE EQUATIONS IN ONE SPATIAL DIMENSION
}

\author{
T. CAZENAVE AND A. HARAUX
}

\begin{abstract}
Let $g$ be a nonincreasing, odd $C^{\mathrm{i}}$ function and $l>0$. We establish that for any solution $u \in C\left(\mathbf{R} ; H_{0}^{1}(0, l)\right)$ of the equation $u_{t t}-u_{x x}+$ $g(u)=0$ and any $\left.x_{0} \in\right] 0, l\left[\right.$, the function $t \mapsto u\left(t, x_{0}\right)$ satisfies the following alternative:

either $u\left(t, x_{0}\right)=0, \forall t \in \mathbf{R}$,

or $\forall a \in \mathbf{R}$, there exist $t_{1}$ and $t_{2}$ in $\left[a, a+2 l_{]}\right.$such that $u\left(t_{1}, x_{0}\right)>0$ and $u\left(t_{2}, x_{0}\right)<0$.

We study the structure of the set of points satisf.ing the first possibility. We give analogous results for $u_{x}$ and for some other homogeneous boundary conditions.
\end{abstract}

0. Introduction. Throughout this paper, $\Omega$ denotes an open, bounded interval of $\mathbf{R}$, and we are studying the oscillatory properties of the general solution of the nonlinear equation

$$
u_{t t}-u_{x x}+g(t, x, u)=0 \text { in } D^{\prime}(J \times \Omega)
$$

where $J$ is a (generally open) interval of $\mathbf{R}$ and $u(t, x)$ satisfies boundary conditions of homogeneous type (namely Dirichlet, Neumann or periodic boundary conditions).

The results that we obtain are of local type, which means that we are studying the function $t \mapsto u(t, x)$ (respectively $u_{x}(t, x)$ ) where $x \in \Omega$ is fixed. While global results on the sign of the function $t \mapsto u(t, \cdot)$ are rather easy to obtain in any spatial dimension (cf. [2, Theorem 2.1, p. 449]), results of local type are more difficult and in fact, even in the case of one-dimensional $\Omega$ the situation will appear somewhat complicated. However, all the proofs of the main results rely on the following simple ideas:

- The solutions $u$ will be extended on $J \times \mathbf{R}$ by symmetry and periodicity considerations, in order to avoid "reflection of the characteristics against the boundary".

-Assuming for example that $u$ and $u_{x}$ are nonnegative on $J \times\left\{x_{0}\right\}$, under simple hypotheses on $g$ which are sufficient to imply the oscillatory character of all solutions, the above extension $\tilde{u}$ will appear to be $\geq 0$ in a characteristic triangle with basis $J \times\left\{x_{0}\right\}$. On replacing $\tilde{u}$ by its even part with respect to $x_{0}$, we shall therefore obtain that the condition " $u$ nonnegative on $J \times\left\{\hat{z}_{0}\right\}$ " implies severe restrictions on the solution if $J$ is large enough.

The main results of this paper are variants of Theorem 2.2.1 which has been announced in [2]. Here we use the same method to study the oscillations of $u_{x}$ and

Received by the editors February 20, 1985.

1980 Mathematics Subject Classification (1985 Revision). Primary 35L05, 35B05, 35R45; Secondary 35B40, 35B60, 34C15. 
the oscillations of $u$ in the case of periodic or Neumann boundary conditions. In order to be self-contained, we stucly the solutions of the semilinear problem and the extension properties of solution s within a framework rather independent of the nonlinear semigroup theory. We : also clarify as much as possible the oscillatory behavior of the solutions to linear equations of the form

$$
u_{t t}-u_{x x}+h(t, x) u=0
$$

with homogeneous boundary corıditions. This preliminary study is useful for two reasons:

-It appears as a step towalids positive oscillation theorems in the semilinear case.

- Counterexamples in the li near case explain why most proofs of our nonlinear oscillation results had to be rat;her involved.

Our work is divided into 3 sections: In $\S 1$, we present all the basic tools which will be used in the proofs of the main results. In $\S 2$, we establish the main oscillation theorems, concerning linear sor semilinear wave equations with Dirichlet boundary conditions. In $\S 3$, the same principles are used to study the other boundary conditions and the oscillations of $u_{x}$ in the three cases. We conclude this section (and the paper) by a few remarks about the possible extensions of our methods and results.

1. Preliminary results. In this section, we have collected a few basic properties of the one-dimensional perturbed wave equations which will be used throughout the paper.

1.1. The inhomogeneous wave equation in a characteristic triangle. Let $a$ be a positive real number. We set

$$
\begin{aligned}
& T=\left\{(t, x) \in \mathbf{R}^{2}, t>0, t+|x|<a\right\}, \\
& R=\left\{(t, x) \in \mathbf{R}^{2}, 0<t<a,-a<x<a\right\} .
\end{aligned}
$$

We define $P \in \mathcal{L}\left(L^{2}(\tau), L^{2}(R)\right)$ by

$$
P u= \begin{cases}u & \text { in } \tau \\ 0 & \text { in } R \backslash \tau .\end{cases}
$$

And finally we considier the functional class

$$
W=\left\{u \in H^{1}(\tau),\left(P u, P\left(u_{t}\right), P\left(u_{x}\right)\right) \in C\left(\left[0, a\left[,\left[L^{2}(-a, a)\right]^{3}\right)\right\} .\right.\right.
$$

We now consider any function $f \in L^{2}(\tau)$ and the inhomogeneous equation

$$
u_{t t}-u_{x x}=f(t, x), \quad(t, x) \in \tau \text {. }
$$

Proposition 1.. 1. Let the functions $(f, u)$ be such that $f \in L^{2}(\tau), u \in$ $H^{1}(\tau)$. Then the following properties are equivalent:

(a) The function $u$ satisfies (1.1.1) in the sense of $D^{\prime}(T)$.

(b) We have $u \in W$ and $u$ is given in $\tau$ by the formula

$$
\begin{aligned}
u(t, x)= & \frac{1}{2}\left\{u_{0}(x+t)+u_{0}(x-t)\right\}+\frac{1}{2} \int_{x-t}^{x+t} v_{0}(s) d s \\
& +\frac{1}{2} \int_{0}^{t} d s \int_{x-(t-s)}^{x+t-s} f(s, \sigma) d \sigma
\end{aligned}
$$


with

$$
u_{0}(x)=u(0, x), \quad v_{0}(x)=u_{t}(0, x) .
$$

ProOF OF PROPOSITION 1.1. The proof will be carried out in several steps.

Step 1. Let $\varphi, \psi$ be two functions in $H^{1}(-a, a)$. Then the function

$$
w(t, x)=\varphi(x+t)+\psi(x-t)+\frac{1}{2} \int_{0}^{t} d s \int_{x-(t-s)}^{x+t-s} f(s, \sigma) d \sigma
$$

is a solution of (1.1.1) in $D^{\prime}(\tau)$ and we have $w \in C(\bar{\tau})$ with in addition

$$
\begin{array}{ll}
\lim _{t \rightarrow 0}(P w)(t, x)=\varphi(x)+\psi(x) & \text { in } L^{2}(-a, a), \\
\lim _{t \rightarrow 0}\left(P w_{x}\right)(t, x)=\varphi^{\prime}(x)+\psi^{\prime}(x) & \text { in } L^{2}(-a, a), \\
\lim _{t \rightarrow 0}\left(P w_{t}\right)(t, x)=\varphi^{\prime}(x)-\psi^{\prime}(x) & \text { in } L^{2}(-a, a) .
\end{array}
$$

Proof. We consider first the case where $\varphi, \psi$ and $f$ are $C^{\infty}$ : then $w$ is also $C^{\infty}$ in $\tau$ and we have the formulas

$$
\begin{aligned}
& w_{x}(t, x)=\varphi^{\prime}(x+t)+\psi^{\prime}(x-t)+\frac{1}{2} \int_{0}^{t}[f(s, x+t-s)+f(s, s-t+s)] d s \\
& w_{t}(t, x)=\varphi^{\prime}(x+t)-\psi^{\prime}(x-t)+\frac{1}{2} \int_{0}^{t}[f(s, x+t-s)-f(s, x-t+s)] d s
\end{aligned}
$$

By computing $w_{t t}$ and $w_{x x}$ from (1.1.6) and taking the difference we obtain at once that $w$ satisfies (1.1.1). Since on the other hand the function $w$ defined by (1.1.4) depends linearly on $(\varphi, \psi, f)$ and the operator $(\varphi, \psi, f) \mapsto w$ is bounded from $\left[H^{1}(-a, a)\right]^{2} \times L^{2}(\tau)$ to $H^{1}(\tau) \cap L^{\infty}(\tau)$ as a consequence of formulas (1.1.4) and (1.1.6), it is easy to check that (1.1.1) is satisfied in the general case as well as formulas (1.1.6). Finally it is easy to see that (1.1.5) is satisfied when $f=0$. Thus all we need to do is to check (1.1.5) when $\varphi=\psi=0, f \neq 0$. But in that case it is rather straightforward to deduce that the $L^{2}$-norms of the 3 functions $(P w)(t, x)$, $\left(P w_{x}\right)(t, x)$ and $\left(P w_{t}\right)(t, x)$ are bounded by $C t^{1 / 2}$ for some finite constant $C$. Hence the proof of Step 1 is achieved.

Step 2. Let $u \in H^{1}(\tau)$ be any solution of (1.1.1) in $D^{\prime}(\tau)$. Then there exists $\varphi, \psi$ in $H^{1}(-a, a)$ such that $u(t, x)$ is given by the right-hand side of (1.1.4).

PROOF. We introduce

$$
\tilde{u}(t, x)=u(t, x)-\frac{1}{2} \int_{0}^{t} d s \int_{x-(t-s)}^{x+t-s} f(s, \sigma) d \sigma .
$$

Since $u$ is a solution of (1.1) we have

$$
\tilde{u}_{t t}-\tilde{u}_{x x}=0 \text { in } D^{\prime}(\tau) \text {. }
$$

By setting $\xi=x+t, \eta=x-t$ and $v(\xi, \eta)=\tilde{u}(t, x)=\tilde{u}((\xi-\eta) / 2,(\xi+\eta) / 2)$, we obtain that $v$ is a solution of

with

$$
\frac{\partial^{2} v}{\partial \xi \partial \eta}=0 \text { in } D^{\prime}(\mathcal{U})
$$

$$
\mathcal{U}=\left\{(\xi, \eta) \in \mathbf{R}^{2}, \xi>\eta \text { and } \operatorname{Sup}\{|\xi|,|\eta|\}<a\right\}
$$


It can be easily deduced from the standard theory of distributions that $v(\xi, \eta)$ must be a function of the form $\varphi(\xi)+\psi(\eta)$ with $\varphi, \psi$ in $H^{1}(-a, a)$.

Now coming back to the variables $(t, x)$ we get $\tilde{u}(t, x)=\varphi(x+t)+\psi(x-t)$, hence the proof of Step 2 is achieved.

Step 3. Let $u$ be as in Step 2. Then in fact we have $u \in W$ and formulas (1.1.2) and (1.1.3) are satisfied.

PROOF. As a consequence of Steps 1 and 2 we know that $u(t, x)$ and $u_{t}(t, x)$ have a limit as $t \rightarrow 0(t>0)$ in the strong topology of $L^{2}(-a+\varepsilon, a-\varepsilon)$ for any $\varepsilon>0$. Moreover formulas (1.1.4) and (1.1.6) are valid at $t=0$, hence

$$
u(0, x)=\varphi(x)+\psi(x), \quad u_{t}(0, x)=\varphi^{\prime}(x)-\psi^{\prime}(x) .
$$

From (1.1.8) it is easy to deduce that for any $(t, x) \in \tau$, we have

$$
\varphi(x+t)+\psi(x-t)=\frac{1}{2}\left\{u_{0}(x+t)+u_{0}(x-t)\right\}+\frac{1}{2} \int_{x-t}^{x+t} v_{0}(s) d s
$$

with $u_{0}, v_{0}$ given by (1.1.3). Hence we have proved (1.1.2) and (1.1.3).

Now in order to prove that $u \in W$, we consider $\bar{T}^{\prime}=\left\{(t, x) \in \mathbf{R}^{2}, t \leq 0\right.$, $|t|+|x|<a\}$ and we extend $\tilde{u}$ in $C=\tau \cup \bar{\tau}^{\prime}$ by

$$
\tilde{u}(t, x)=\varphi(x+t)+\psi(x-t)+\frac{1}{2} \int_{0}^{t^{+}} d s \int_{x-(t-s)}^{x+t-s} f(s, \sigma) d \sigma .
$$

Then $\tilde{u} \in H^{1}(C)$ and $\tilde{u}_{t t}-\tilde{u}_{x x}=\tilde{f}$ in $D^{\prime}(\tau)$ with

$$
\tilde{f}= \begin{cases}f & \text { in } T \\ 0 & \text { in } \bar{T}^{\prime}\end{cases}
$$

Finally, we apply (1.1.5) to the restriction of $\tilde{u}$ to any characteristic (forward or backward) triangle with basis $\{t=\rho,|x|<a-\rho\}$ with $0<\rho<a$.

1.2. Boundary value problems and extension properties. In this section we will use the following notation: $a, b, t_{1}, t_{2}$ are real numbers such that $a<b, t_{1}<t_{2}$ and we set

$$
J=] t_{1}, t_{2}[, \quad \Omega=] a, b[, \quad Q=J \times \Omega .
$$

An important consequence of Proposition 1.1 is that solutions of the wave equation in $Q$ have some regularity properties up to the boundary $\partial Q=(\partial J \times \Omega) \cup$ $(J \times \partial \Omega)$. More precisely, for any $t \in\left[t_{1}, t_{2}\right]$ we can define $u(t, \cdot) \in H^{1}(\Omega)$ and $u_{t}(t, \cdot) \in L^{2}(\Omega)$ where $u \in H^{1}(Q)$ is any solution of $u_{t t}-u_{x x}=f$ in $D^{\prime}(Q)$ with $f \in L^{2}(Q)$.

For such a function $u$ we can define as well $u(\cdot, x) \in H^{1}(J)$ and $u_{x}(\cdot, x) \in L^{2}(J)$ for any $x \in[a, b]$.

This last property is convenient to define the homogeneous boundary value problems associated with the inhomogeneous wave equations in $Q$.

DEFINITION 1.2.1. Let $u \in H^{1}(Q)$ and $f \in L^{2}(Q)$ be such that $u_{t t}-u_{x x}=f$ in $D^{\prime}(Q)$. We say that $u$ satisfies the periodic (respectively Neumann; Dirichlet) boundary conditions in $\Omega$ if $u(t, a)=u(t, b)$ and $u_{x}(t, a)=u_{x}(t, b)$ a.e. on $J$ [resp. $\left.u_{x}(t, a)=u_{x}(t, b)=0 ; u(t, a)=u(t, b)=0\right]$.

An important tool which will be used in $\S \S 2$ and 3 is the extension of a solution of the wave equation with one of the boundary conditions above to a solution in a strip $J \times \mathbf{R}$ which is periodic with respect to $x$. More precisely, we have the following results. 
LeMma 1.2.2. Let $u$ and $f$ be as in Definition 1.2.1 and assume $a=0, b=l$. We define $\tilde{u}$ and $\tilde{f}$ in $L_{\text {loc }}^{2}(J \times \mathbf{R})$ as follows:

-If $u$ satisfies the periodic boundary conditions in $\Omega, \tilde{u}$ and $\tilde{f}$ are respectively the unique extensions of $u$ and $f$ which are l-periodic with respect to $x$ in $J \times \mathbf{R}$.

-If $u$ satisfies the Neumann boundary conditions in $\Omega, \tilde{u}$ and $\tilde{f}$ are respectively the unique extensions of $u$ and $f$ which are even and $2 l$-periodic with respect to $x$ in $J \times \mathbf{R}$.

-If $u$ satisfies the Dirichlet boundary conditions in $\Omega, \tilde{u}$ and $\tilde{f}$ are respectively the unique extensions of $u$ and $f$ which are odd and 2l-periodic with respect to $x$ in $J \times \mathbf{R}$.

Then we have: $\tilde{u}_{t t}-\tilde{u}_{x x}=\tilde{f}$ in $D^{\prime}(J \times \mathbf{R})$ (and $\tilde{u}$ satisfies the 2 -periodic boundary conditions in $] 0,2 l[)$.

Proof. We only give it in the case of periodic boundary conditions since the other cases are quite similar. Since $\tilde{u}$ and $\tilde{f}$ are $l$-periodic in $x$ it is sufficient to establish that we have

$$
\tilde{u}_{t t}-\tilde{u}_{x x}=\tilde{f}(t, x) \quad \text { in } D^{\prime}(J \times]-l, l[) .
$$

Also clearly we can assume that $|J|<l$ and $J$ is symmetric with respect to 0 .

We now introduce three open subsets of $J \times]-l, l[$ :

$$
\begin{gathered}
\left.R^{+}=J \times\right] 0, l\left[, \quad R^{-}=J \times\right]-l, 0[, \\
C=\{(t, x) \in J \times]-l, l[,|t|+|x|<|J|\} .
\end{gathered}
$$

Since the equation is obviously satisfied in $R^{+}$and $R^{-}$and $R^{+} \cup R^{-} \cup C=$ $J \times]-l, l[$, the whole matter is reduced to checking that we have

$$
\tilde{u}_{t t}-\tilde{u}_{x x}=\tilde{f} \text { in } D^{\prime}(C) \text {. }
$$

Since the equation is satisfied in $R^{+}$, hence in $C \cap\{x>0\}=\tau$, for any $(t, x) \in \tau$ we have

$$
\begin{aligned}
\tilde{u}(t, x)= & \frac{1}{2}\left\{\tilde{u}_{0}(t+x)+\tilde{u}_{0}(t-x)\right\}+\frac{1}{2} \int_{t-x}^{t+x} \tilde{v}_{0}(s) d s \\
& -\frac{1}{2} \int_{0}^{x} d \sigma \int_{t-(x-\sigma)}^{t+(x-\sigma)} \tilde{f}(s, \sigma) d s
\end{aligned}
$$

with $\tilde{u}_{0}(\cdot)=u(\cdot, 0)$ and $\tilde{v}_{0}(\cdot)=u_{x}(\cdot, 0)$.

Now let $\tau_{1}=\{(t, x) \in J \times] 0, l[,|t|+l-x \leq|J|\}$.

As a consequence of Proposition 1.1, since $\tilde{u}(t, l-x)=\tilde{w}(t, x)$ is a solution of $\tilde{w}_{t t}-\tilde{w}_{x x}=\tilde{f}(t, l-x)$ in $\tau$, we have

$$
\begin{aligned}
\forall(t, x) \in \tau, \quad \tilde{w}(t, x)= & \frac{1}{2}\left\{\tilde{w}_{0}(t+x)+\tilde{w}_{0}(t-x)\right\}+\frac{1}{2} \int_{t-x}^{t+x} \tilde{z}_{0}(s) d s \\
& -\frac{1}{2} \int_{0}^{x} d \sigma \int_{t-(x+\sigma)}^{t+(x-\sigma)} \tilde{f}(s, l-\sigma) d s
\end{aligned}
$$

with

$$
\begin{aligned}
& \tilde{w}_{0}(\cdot)=\tilde{w}(\cdot, 0)=\tilde{u}(\cdot, l)=\tilde{u}(\cdot, 0)=\tilde{u}_{0}(\cdot) \\
& \tilde{z}_{0}(\cdot)=\tilde{w}_{x}(\cdot, 0)=-\tilde{u}_{x}(\cdot, l)=-\tilde{u}_{x}(\cdot, 0)=-\tilde{v}_{0}(\cdot) .
\end{aligned}
$$


This implies immediately

$$
\begin{aligned}
\tilde{u}(t, x)= & \tilde{w}(t, l-x)=\frac{1}{2}\left\{\tilde{u}_{0}(t+l-x)+\tilde{u}_{0}(t-l+x)\right\} \\
& -\frac{1}{2} \int_{t-l+x}^{t+l-x} \tilde{v}_{0}(s) d s-\frac{1}{2} \int_{0}^{l-x} d \sigma \int_{t-(l-x-\sigma)}^{t+l-x-\sigma} \tilde{f}(s, l-\sigma) d s, \quad \forall(t, x) \in \tau_{1} .
\end{aligned}
$$

Finally, by setting $x-l=y \in]-l, 0[$ we find

$$
\begin{aligned}
\tilde{u}(t, y)= & \frac{1}{2}\left\{\tilde{u}_{0}(t-y)+\tilde{u}_{0}(t+y)\right\}+\frac{1}{2} \int_{t-y}^{t+y} \tilde{v}_{0}(s) d s \\
& -\frac{1}{2} \int_{0}^{y} d \sigma \int_{t-(y-\sigma)}^{t+y-\sigma} \tilde{f}(s, \sigma) d s
\end{aligned}
$$

for all $(t, y) \in \tau^{\prime}=\{(t, y) \in J \times]-l, 0[,|t|-y \leq|J|\}$. Hence (1.2.1) is in fact valid for any $(t, x) \in C$. The end of the proof is identical to that of Step 1 of Proposition 1.1 .

1.3. The sign of solutions in a characteristic triangle. In this section, we introduce a simple idea which will turn out to be fundamental in the study of the oscillation properties of solutions to semilinear wave equations with homogeneous boundary conditions in one space dimension.

We start with a very simple observation.

PROPOSITION 1.3.1. Let $u$ satisfy (1.1.1) in $T$ and assume that we have

$$
f(t, x) \geq 0 \quad \text { a.e. in } T \text {. }
$$

Then we have

$$
u(t, x) \geq \frac{1}{2}\left\{u_{0}(x+t)+u_{0}(x-t)\right\}+\frac{1}{2} \int_{x-t}^{x+t} v_{0}(s) d s \quad \text { for all }(t, x) \in \tau .
$$

As a particular case, if $u_{0} \geq 0$ and $v_{0} \geq 0$ on $[-a, a]$, then $u \geq 0$ in $\tau$.

PROOF. Immediate consequence of formula (1.1.2).

Now we turn to a more subtle property in the same vein which has to do with "positivity preserving" for some linear perturbations of the wave equation.

From now on we consider $a>0, \tau$ as in $\S 1.1$ and a function $h \in L^{\infty}(\tau)$ such that

$$
h(t, x) \geq 0 \text { a.e. in } \tau .
$$

LEMMA 1.3.2. Let $u \in H^{1}(\tau)$ be a solution of the equation

$$
u_{t t}-u_{x x}=h(t, x) u(t, x) \quad \text { in } D^{\prime}(\tau)
$$

with $h \in L^{\infty}(\tau)$ satisfying (1.3.3).

If in addition we assume

$$
u_{0} \geq 0 \text { and } v_{0} \geq 0 \text { a.e. on }[-a, a]
$$

then we have

$$
u \geq 0 \text { a.e. on } \tau
$$

and more precisely $u$ satisfies (1.3.2). 
Proof. As a consequence of formula (1.1.2), for all $(t, x) \in \tau$ we have

$$
\begin{aligned}
u(t, x) & \geq \frac{1}{2} \int_{0}^{t} d s \int_{x-(t-s)}^{x+t-s} h(s, \sigma) u(s, \sigma) d \sigma \\
& \geq-\frac{1}{2} \int_{0}^{t} d s \int_{x-(t-s)}^{x+t-s} h(s, \sigma) u^{-}(s, \sigma) d \sigma, \quad \text { since } h \geq 0 .
\end{aligned}
$$

The last inequality is equivalent to

$$
-u(t, x) \leq \frac{1}{2} \int_{0}^{t} d s \int_{x-(t-s)}^{x+t-s} h(s, \sigma) u^{-}(s, \sigma) d \sigma .
$$

Since the right-hand side is $\geq 0$, we obtain

$$
\forall(t, x) \in \tau, \quad u^{-}(t, x) \leq \frac{1}{2} \int_{0}^{t} d s \int_{x-(t-s)}^{x+t-s} h(s, \sigma) u^{-}(s, \sigma) d \sigma .
$$

Let $M=\frac{1}{2} \operatorname{Supess}_{(t, x) \in C}|h(t, x)|$. From (1.3.7) we deduce with $u^{-}(t, x)=$ $w(t, x)$ :

$$
\forall(t, x) \in \tau, \quad w(t, x) \leq M \int_{0}^{t} \int_{x-(t-s)}^{x+t-s} w(s, \sigma) d \sigma d s .
$$

Since $t+|x| \leq a$ and $t \geq 0$, we have for all $s \in[0, t]$ the inequalities

$$
-a+s \leq x-t+s \leq x+t-s \leq a-s .
$$

Hence from (1.18) we deduce at once

$$
w(t, x) \leq M \int_{0}^{t} \int_{-a+s}^{a-s} w(s, \sigma) d \sigma d s
$$

The right-hand side in (1.3.9) does not depend on $x$.

By integrating both sides of $(1.3 .9)$ on $[-a+t, a-t]$ with respect to $x$, we obtain

$$
\psi(t) \leq 2 M a \int_{0}^{t} \psi(s) d s
$$

with

$$
\psi(t)=\int_{-a+t}^{a-t} w(t, x) d x, \quad \forall t \in[0, a[.
$$

Finally

$$
\psi(t) \leq \exp (2 M a) \psi(0)=0, \quad \forall t \in[0, a[.
$$

From $\psi(t)=0$ on $[0, a$ [ we deduce immediately that (1.3.6) is satisfied. Then it suffices to apply Proposition 1.3.1 with $f=h \cdot u$ to obtain (1.3.2).

REMARK 1.3.3. (a) The results of Proposition 1.3.1 and Lemma 1.3.2 will be our main tools to study the oscillation properties of the solutions to semilinear wave equations with homogeneous boundary conditions in $\Omega=] 0, l[$.

(b) For the sake of completeness we now recall some basic results on local existence and uniqueness for semilinear wave equations in $\Omega$, and we specify some additional regularity properties which will be convenient later on. 
1.4. Local existence, uniqueness and regularity for semilinear one dimensional wave equations. In this section we set $\Omega=] 0, l[$ with $l>0 . J$ is an open interval such that $0 \in J$.

We consider a function $f: J \times \Omega \times \mathbf{R} \rightarrow \mathbf{R}$ as follows:

$-f$ is integrable with respect to $(t, x) \in J \times \Omega$, uniformly with respect to bounded values of $u$.

$-f$ is locally lipschitz-continuous with respect to $u \in \mathbf{R}$, uniformly with respect to $(t, x) \in J \times \Omega$.

We are looking for a function $u \in H^{1}(J \times \Omega)$ which would satisfy the following properties:

(1.4.1) (a) $u_{t t}-u_{x x}+f(t, x, u(t, x))=\Omega$ in $D^{\prime}(J \times \Omega)$.

(b) $u$ satisfies one of the usual homogeneous boundary conditions (periodic, Dirichlet or Neumann, cf. Definition 1.2.1).

(c) $u(0, x)=u_{0}(x)$ and $u_{t}(0, x)=v_{0}(x)$ with $u_{0} \in H^{1}(\Omega)$ and $v_{0} \in L^{2}(\Omega)$ given functions.

According to the results of $\S \S 1.1$ and 1.2 , conditions (b) and (c) make sense as soon as (a) is fulfilled.

On the other hand, by using the techniques of [8], it is possible to show that for any $\left(u_{0}, v_{0}\right) \in H^{1}(\Omega) \times L^{2}(\Omega)$ satisfying those of the boundary conditions which make sense, i.e.

$u_{0}(0)=u_{0}(l)=0$ in the case of Dirichlet conditions,

$u_{0}(0)=u_{0}(l)$ in the case of periodic conditions,

no condition at all in the case of Neumann conditions,

there exists $J$, depending on $\left(u_{0}, v_{0}\right)$ in the general case and a unique function $u$ which fulfills (a)-(c).

In addition, $u$ satisfies the following regularity property:

$$
\begin{aligned}
& \forall(\alpha, \beta) \in \mathbf{R}^{2} \text { such that } \alpha<\beta \text { and }[\alpha, \beta] \subset J, \\
& u \in C\left([\alpha, \beta] ; H^{1}(\Omega)\right) \cap C^{1}\left([\alpha, \beta] ; L^{2}(\Omega)\right) .
\end{aligned}
$$

It is more or less standard to remark that (1.4.2) implies $u \in C([\alpha, \beta] \times \bar{\Omega})$, and this regularity property is consistent with the regularity of solutions of the wave equation in a characteristic triangle.

In fact, property (1.4.2) implies more than that, as shown by the following simple remark.

LEMMA 1.4.1. We have for any $(\alpha, \beta)$ and $\Omega$ as above

$$
L^{\infty}\left(\alpha, \beta, H^{1}(\Omega)\right) \cap W^{1, \infty}\left(\alpha, \beta, L^{2}(\Omega)\right) \hookrightarrow C^{0,1 / 2}(\bar{Q})
$$

with $\bar{Q}=[\alpha, \beta] \times \bar{\Omega}$.

ProOF. Let $X=L^{\infty}\left(\alpha, \beta, H^{1}(\Omega)\right) \cap W^{1, \infty}\left(\alpha, \beta, L^{2}(\Omega)\right)$. We only need to show that for any $u \in C^{\infty}(\bar{Q})$, the norm of $u$ in $C^{0,1 / 2}(\bar{Q})$ remains bounded when the norm of $u$ in $X$ stays $\leq 1$. 
For $u \in C^{\infty}(\bar{Q})$ with $\|u\|_{X} \leq 1$, we have

$$
\begin{gathered}
\forall(t, \theta) \in[a, \beta]^{2}, \quad \forall(x, y) \in \bar{\Omega} \times \bar{\Omega}, \\
|u(t, x)-u(\theta, y)| \leq|u(t, x)-u(\theta, x)|+C_{1}|x-y|^{1 / 2}
\end{gathered}
$$

since $H^{1}(\Omega) \hookrightarrow C^{0,1 / 2}(\bar{\Omega})$.

On the other hand, let $x$ be now fixed in $\bar{\Omega}$ and set $B_{\varepsilon}=\bar{B}(x, \varepsilon) \cap \bar{\Omega}$. Then for all $t \in[\alpha, \beta]$, we have

$$
\left|u(t, x)-\frac{1}{\left|B_{\varepsilon}\right|} \int_{B_{\varepsilon}} u(t, \xi) d \xi\right| \leq C_{2} \varepsilon^{1 / 2}
$$

for the same reason as previously.

From (1.4.4) and (1.4.5) we now deduce

$$
|u(t, x)-u(\theta, y)| \leq C_{1}|x-y|^{1 / 2}+C_{2} \varepsilon^{1 / 2}+\frac{1}{\left|B_{\varepsilon}\right|} \int_{B_{\varepsilon}}|u(t, \xi)-u(\theta, \xi)| d \xi
$$

Now

$$
\int_{B_{\varepsilon}}|u(t, \xi)-u(\theta, \xi)| d \xi \leq\left|B_{\varepsilon}\right|^{1 / 2}|u(t, \cdot)-u(\theta, \cdot)|_{L^{2}(\Omega)} .
$$

Thus we obtain, since $u$ is bounded in $W^{1, \infty}\left(\alpha, \beta, L^{2}(\Omega)\right)$

$$
\frac{1}{\left|B_{\varepsilon}\right|} \int_{B_{\varepsilon}}|u(t, \xi)-u(\theta, \xi)| d \xi \leq C_{3}\left|B_{\varepsilon}\right|^{-1 / 2}|t-\theta| \text {. }
$$

Finally, we have $\varepsilon / 2 \leq\left|B_{\varepsilon}\right| \leq \varepsilon$ as soon as $\varepsilon<\beta-\alpha$. Thus from (1.4.6) and (1.4.7) we deduce

$$
|u(t, x)-u(\theta, y)| \leq C_{1}|x-y|^{1 / 2}+C_{2} \varepsilon^{1 / 2}+C_{4} \varepsilon^{-1 / 2}|t-\theta| .
$$

For $|x-y|$ and $|t-\theta|$ small enough we may choose $\varepsilon=|t-\theta|$ (if $t=\theta$ there is no problem). Hence the proof of Lemma 1.4.1 is completed.

REMARK 1.4.2. The embedding property (1.4.3) implies that any solution of a one-dimensional problem as above in the "energy space" $H^{1}(\Omega) \times L^{2}(\Omega)$ is in fact in $C^{0,1 / 2}([\alpha, \beta] \times \bar{\Omega})$ for any compact interval $[\alpha, \beta]$ contained in the maximal interval of existence.

Moreover, those solutions which are global and such that

$$
u \in L^{\infty}\left(\mathbf{R}, H^{1}(\Omega)\right) \cap W^{1, \infty}\left(\mathbf{R}, L^{2}(\Omega)\right)
$$

are in fact globally Hölder-continuous with exponent $\frac{1}{2}$ in $\mathbf{R} \times \bar{\Omega}$.

This property will be used to ease the treatment of asymptotic behavior in the case of autonomous equations with the "good sign" (cf. §2).

REMARK 1.4.3. In this one-dimensional context, a lot of things can be done in the phase space $W^{1, p}(\Omega) \times L^{p}(\Omega)$ with $p>1$. But the "energy space" is the only one which allows us to obtain uniform bounds for $t \geq 0$ when we consider semilinear problems. Also the possibility of working with $p \neq 2$ will disappear completely in dimensions $>1$. For these reasons we have written all these preliminaries in a Hilbert space setting, which also makes the statements simpler. 
2. The case of Dirichlet boundary conditions. Let $l>0, \Omega=] 0, l[$ and $J \subset \mathbf{R}$ be an open interval. In this section, we study oscillatory properties of local (with respect to space) type for the solutions of some perturbed wave equations in $Q=J \times \Omega$ with Dirichlet boundary conditions on $J \times \partial \Omega$.

2.1. Some oscillation results for linear equations in $Q$. Let us start with a simple property which is true under very general conditions.

THEOREM 2.1.1. Let $h \in L^{\infty}(Q)$ be nonnegative and let $u \in H^{1}(Q)$ be a solution of

$$
\begin{aligned}
& u_{t t}-u_{x x}+h(t, x) u=0 \quad \text { in } D^{\prime}(Q), \\
& u(t, 0)=u(t, l)=0, \quad \forall t \in J .
\end{aligned}
$$

Assume that $|J| \geq 2 l$ and $u \not \equiv 0$ in $Q$. Then we have

$$
\begin{aligned}
& \operatorname{meas}\left\{t \in J, u_{x}(t, 0)>0\right\}>0, \\
& \operatorname{meas}\left\{t \in J, u_{x}(t, 0)<0\right\}>0 .
\end{aligned}
$$

Proof OF THEOREM 2.1.1. From (2.1.1) we deduce $u_{x x}-u_{t t}=h(t, x) u$ in $D^{\prime}(Q)$. Assume that (2.1.2) is not satisfied, with for example: $u_{x}(t, 0) \geq 0$ a.e. on $J,|J| \geq 2 l$ and $u \neq \equiv$ in $Q$.

We consider the triangle

$$
T=\left\{(t, x) \in Q, x+\left|t-t_{0}\right|<l\right\}
$$

where $t_{0}$ is some point such that $] t_{0}-l, t_{0}+l[\subset J$.

By exchanging the roles of $t$ and $x$, the application of Lemma 1.3.2 gives that $u \geq 0$ in $T$ and moreover

$$
u(t, x) \geq \frac{1}{2} \int_{t-x}^{t+x} u_{x}(s, 0) d s, \quad \forall(t, x) \in T .
$$

Now in (2.1.3) we can let $(t, x) \rightarrow\left(t_{0}, l\right)$. We obtain

$$
0=u\left(t_{0}, l\right) \geq \frac{1}{2} \int_{t_{0}-l}^{t_{0}+l} u_{x}(s, 0) d s .
$$

It is obvious that (2.1.4) implies

$$
\left.u_{x}(t, 0) \equiv 0 \quad \text { a.e. on }\right] t_{0}-l, t_{0}+l[\text {. }
$$

Then by changing $u$ to $(-u)$, the argument just above gives

$$
u(t, x) \equiv 0, \quad \forall(t, x) \in T .
$$

As an immediate consequence we have

$$
u\left(t_{0}, x\right)=u_{t}\left(t_{0}, x\right)=0 \quad \text { a.e. in } \Omega .
$$

Since $h \in L^{\infty}(Q)$, from (2.1.1) and (2.1.6) we conclude that $u \equiv 0$ in $Q$ (by uniqueness of the initial value problem for (2.1.1)).

REMARK 2.1.2. If $u$ is a "regular" solution of (2.1.1) Theorem 2.1.1 allows us to deduce that $u(t, x)$ "oscillates" as $t$ ranges over $J$ for all $x$ close enough to the boundary $\partial \Omega=\{0, l\}$. In the special case where $h \in L^{\infty}(\mathbf{R} \times \Omega)$ and $h$ does not depend on $t$, all the solutions of (2.1.1) are almost periodic in the energy space. Then the oscillations are "transmitted" to almost all points $x \in \Omega$, as shown by the following result. 
THEOREM 2.1.3. Let $\rho \in L^{\infty}(\Omega)$ with $\rho \geq 0$, and let $u(t, x)$ be a solution of

$$
\begin{aligned}
& u \in C\left(\mathbf{R}, H_{0}^{1}(\Omega)\right) \cap C^{1}\left(\mathbf{R}, L^{2}(\Omega)\right), \\
& u_{t t}-u_{x x}+\rho(x) u(t, x)=0 \quad \text { in } D^{\prime}(\mathbf{R} \times \Omega) .
\end{aligned}
$$

Then for any $x \in \bar{\Omega}$ we have one of the following properties:

$$
\forall t \in \mathbf{R}, \quad u(t, x)=0 .
$$

(2.1.9) $u(t, x)$ takes both positive and negative values on any time interval $J$ such that $|J| \geq 2 l$.

PROOF. The unbounded linear operator $A$ on $L^{2}(\Omega)$ defined by

$$
D(A)=H^{2}(\Omega) \cap H_{0}^{1}(\Omega), \quad A u=-u_{x x}+\rho(x) u
$$

is selfadjoint, positive with a compact inverse.

If we write $(2.1 .7)$ as a system in $H_{0}^{1}(\Omega) \times L^{2}(\Omega)$ endowed with the norm $\|(u, v)\|=\left[\int_{\Omega}\left\{A u \cdot u+v^{2}\right\} d x\right]^{1 / 2}$, then (2.1.7) generates an isometry group and by using for example [5, Proposition 22, p. 213] we can prove that any solution $u(t, x)$ of (2.1.7) is almost-periodic as a function: $\mathbf{R} \rightarrow H_{0}^{1}(\Omega)$.

More precisely, let $\left\{\lambda_{j}\right\}_{j \in \mathbf{N}}$ be the family of eigenvalues of $A$ in $L^{2}(\Omega)$ and $\left\{\varphi_{j}\right\}_{j \in \mathbf{N}}$ the corresponding orthonormal system of eigenfunctions (it is well known that the $\lambda_{j}$ are simple). The following lemma summarizes the information which will be needed to deduce Theorem 2.1.3 from Theorem 2.1.1.

LEMMA 2.1.4. (a) The numbers $\lambda_{j}>0$ and the functions $\varphi_{j}$ are such that we have

$$
\begin{gathered}
\operatorname{Sup}_{j \in \mathbf{N}}\left\|\varphi_{j}\right\|_{L^{\infty}(\Omega)}<+\infty \\
\exists c>0, C>0 \text { such that } c \leq\left|\varphi_{j}^{\prime}(0)\right| / \sqrt{\lambda_{j}} \leq C, \quad \forall j \in \mathbf{N}, \\
\sum_{j=0}^{+\infty} \frac{1}{\lambda_{j}}<+\infty
\end{gathered}
$$

(b) The general solution of (2.1.7) is of the form

$$
u(t, x)=\sum_{j=0}^{+\infty} w_{j} \cos \left(\sqrt{\lambda_{j}} t+\alpha_{j}\right) \varphi_{j}(x)
$$

where $\alpha_{j}$ and $w_{j}$ are two sequences of real numbers such that

$$
\sum_{j=0}^{+\infty} \lambda_{j} w_{j}^{2}<+\infty
$$

(c) For a solution of (2.1.7) the following conditions are equivalent:

$$
\text { (i) } u(0) \in H^{2}(\Omega) \text { and } u_{t}(0)=v_{0} \in H_{0}^{1}(\Omega) \text {, }
$$

$$
\text { (ii) } \sum_{j=0}^{+\infty} \lambda_{j}^{2} w_{j}^{2}<+\infty \text {. }
$$


Moreover, any solution of (2.1.7) with these properties is in $C^{1}(\mathbf{R} \times \bar{\Omega})$ and satisfies the identity

$$
u_{x}(t, 0)=\sum_{j=0}^{+\infty} w_{j} \varphi_{j}^{\prime}(0) \cos \left(\sqrt{\lambda_{j}} t+\alpha_{j}\right)
$$

where the series on the right-hand side are uniformly convergent on $\mathbf{R}$.

PROOF. (a) By definition of $\varphi_{j}$ we have

$$
\left.\varphi_{j}^{\prime \prime}+\lambda_{j} \varphi_{j}=\rho(x) \varphi_{j} \quad \text { on } \Omega=\right] 0, l[\text {. }
$$

Let $F_{j}(x)=\varphi_{j}^{\prime 2}(x)+\lambda_{j} \varphi_{j}^{2}(x), \forall x \in \Omega$. If we multiply (2.1.18) by $\varphi_{j}^{\prime}$, we find

$$
\begin{gathered}
\frac{d}{d x}\left(F_{j}(x)\right)=2 \rho(x) \varphi_{j}(x) \varphi_{j}^{\prime}(x), \\
\left|\frac{d}{d x}\left(F_{j}(x)\right)\right| \leq C_{1} F_{j}(x), \quad \forall j \in \mathbf{N}, \forall x \in \Omega .
\end{gathered}
$$

As an immediate consequence of (2.1.20), we have

$$
\forall x \in \Omega, \quad \exp \left(-C_{1} l\right) F_{j}(0) \leq F_{j}(x) \leq \exp \left(C_{1} l\right) F_{j}(0) .
$$

On the other hand, by multiplying $(2.1 .18)$ by $\varphi_{j}(x)$ and integrating over $\Omega$, we obtain

$$
\int_{\Omega} \varphi_{j}^{\prime 2}(x) d x=\int_{\Omega}\left[\lambda_{j}-\rho(x)\right] \varphi_{j}^{2}(x) d x \leq \lambda_{j} .
$$

By integrating (2.1.21) over $\Omega$ we obtain

$$
\lambda_{j} \leq \int_{\Omega} F_{j}(x) d x \leq l \exp \left(C_{1} l\right) F_{j}(0)
$$

and also, taking account of (2.1.22):

$$
l \exp \left(-C_{1} l\right) F_{j}(0) \leq \int_{\Omega} F_{j}(x) d x \leq 2 \lambda_{j}
$$

Hence we find the estimates

$$
\varphi_{j}^{\prime 2}(0)=F_{j}(0) \geq l^{-1} \exp \left(-C_{1} l\right) \lambda_{j}
$$

and (by using (2.1.21) once more)

$$
\operatorname{Sup}_{x \in \Omega} F_{j}(x) \leq \frac{2 \lambda_{j}}{l} \exp \left(2 C_{1} l\right) .
$$

These two inequalities clearly imply (2.1.10) and (2.1.11).

To complete the proof of (a), we choose a fixed function $\varsigma \in C^{\infty}(\bar{\Omega})$ such that

$$
\varsigma(0)=1, \quad \varsigma^{\prime}(0)=\varsigma^{\prime}(l)=\varsigma(l)=0 .
$$


Now if we multiply (2.1.18) by $\zeta(x)$ and integrate over $\Omega$, we find that as a consequence of $(2.1 .23)$

$$
\begin{aligned}
& \int_{\Omega} \varphi_{j}^{\prime \prime} \varsigma d x+\lambda_{j} \int_{\Omega} \varphi_{j} \varsigma d x=\int_{\Omega} \rho(x) \varphi_{j} \varsigma d x \\
& \quad \Rightarrow \lambda_{j} \int_{\Omega} \varphi_{j} \varsigma d x=-\varphi_{j}^{\prime}(0)+\int_{\Omega} \rho(x) \varphi_{j} \varsigma d x-\int_{\Omega} \varphi_{j} \varsigma^{\prime \prime} d x \\
& \quad \Rightarrow \lambda_{j}\left|\int_{\Omega} \varphi_{j} \zeta d x\right| \geq c \sqrt{\lambda_{j}}-M
\end{aligned}
$$

with $c, M$ independent of $j \in \mathbf{N}$.

Hence for $j \geq j_{0}$ large enough we find

$$
\lambda_{j}\left|\int_{\Omega} \varphi_{j} \varsigma d x\right| \geq \frac{c}{2} \sqrt{\lambda_{j}} \Rightarrow \frac{c}{2 \sqrt{\lambda_{j}}} \leq\left|\left\langle\varphi_{j}, \varsigma\right\rangle\right| .
$$

From (2.1.24) it is immediate to deduce

$$
\sum_{j \geq j_{0}} \frac{1}{\lambda_{j}} \leq \sum_{j \geq j_{0}} \frac{4}{c^{2}}\left\langle\varphi_{j}, \zeta\right\rangle^{2} \leq \frac{4}{c^{2}} \int_{\Omega}|\zeta|^{2} d x .
$$

Hence (2.1.12) is now proved.

(b) These properties are rather classical. Formulas (2.1.13) and (2.1.17) are obvious when $u_{0}$ and $v_{0}$ are a finite linear combination of the $\varphi_{j}(x)$. In the general case (2.1.13) is easily established by density. Actually the series is uniformly convergent on $\mathbf{R} \times \bar{\Omega}$ as a consequence of (2.1.10) and (2.1.12).

(c) It is rather easy to check that (i) $\Rightarrow$ (ii) from formula (2.1.13) and the definition of $\varphi_{j}(x)$. The converse (ii) $\Rightarrow$ (i) can be proved by using the fact that the system is autonomous and reasoning on $u_{t}$.

Finally, formula (2.1.17) is also established by density on $\left(u_{0}, v_{0}\right)$. Note that properties (2.1.11) and (2.1.12) play a crucial role in giving a pointwise meaning to (2.1.17).

END OF PROOF OF THEOREM 2.1.3.

Step 1. Let $\left\{v_{j}\right\}_{j \in \mathbf{N}}$ be any sequence of reals such that $\sum_{j=0}^{+\infty} \lambda_{j} v_{j}^{2}<+\infty$. Consider

$$
\bar{u}(t, x)=\sum_{j=0}^{+\infty} \frac{v_{j}}{\varphi_{j(0)}^{\prime}} \cos \left(\sqrt{\lambda_{j}} t+\alpha_{j}\right) \varphi_{j}(x),
$$

where the numbers $\left\{\alpha_{j}\right\}$ are arbitrary.

It is clear that $\bar{u} \in C^{1}(\mathbf{R} \times \bar{\Omega})$ is a solution of (2.1.7) and that we have for all $t \in \mathbf{R}$

$$
\bar{u}_{x}(t, 0)=\sum_{j=0}^{+\infty} v_{j} \cos \left(\sqrt{\lambda_{j}} t+\alpha_{j}\right)=f(t) .
$$

As a consequence of Theorem 2.1.1,

-Either on any interval $J$ such that $|J| \geq 2 l, f(t)$ must take some $>0$ and some $<0$ values,

-or $\bar{u} \equiv 0$ on $\mathbf{R} \times \bar{\Omega}$. Then $f \equiv 0$. 
Step 2. Let $u$ be any solution of (2.1.7) and $x_{0} \in \bar{\Omega}$. As a consequence of (2.1.10), (2.1.13) and (2.1.14), the function

$$
f(t)=u\left(t, x_{0}\right)=\sum_{j=0}^{+\infty} w_{j} \cos \left(\sqrt{\lambda_{j}} t+\alpha_{j}\right) \varphi_{j}\left(x_{0}\right)
$$

is of the type considered above with $v_{j}=w_{j} \varphi_{j}\left(x_{0}\right)$.

By using the result of Step 1 applied to $f(t)=u\left(t, x_{0}\right)$, we obtain exactly the alternative saying that either (2.1.8) or (2.1.9) is fulfilled.

Hence the proof of Theorem 2.1.3 is complete.

REMARK 2.1.5. This way of proving Theorem 2.1.3 seems at first sight rather unnatural, and one naturally wonders whether the analogous statement is still true with $\rho(x) \geq 0$ replaced by $h(t, x) \geq 0$ and bounded. We shall see later $(\S 2.2)$ that we can replace $\rho(x)$ by $h(t)$. The proof relies on a quite different argument, and actually the general statement when $h$ depends on both $(t, x)$ is false, as shown by the following counterexample.

EXAMPLE 2.1.6. We take $l=2 \pi$ and

$$
\begin{aligned}
& h(t, x)= \begin{cases}1 & \text { if } t>0, x>\pi \\
0 & \text { otherwise }\end{cases} \\
& u_{0}(x)=\sin x \text { and } v_{0}(x)=0 .
\end{aligned}
$$

It is perfectly clear that here (2.1.1) has a unique solution $u$ such that $u(0)=u_{0}$ and $u_{t}(0)=v_{0}$. Moreover we have

$$
u(t, x)=\sin t \sin x, \quad \forall t \leq 0 .
$$

This formula immediately implies

$$
\forall t \leq 0, \quad u(t, \pi)=0 .
$$

We claim that there does not exist any $\tau>0$ such that $u(t, \pi)=0$ on $[0, \tau]$. Indeed, if such a property was true, we should have, by uniqueness of the Cauchy problem with Dirichlet boundary conditions in $[0, \tau] \times] 0, \pi[$ and $[0, \tau] \times] \pi, 2 \pi[$

$$
\begin{aligned}
& \forall t \in[0, \tau], \forall x \in] 0, \pi[, \quad u(t, x)=\sin t \sin x, \\
& \forall t \in[0, \tau], \forall x \in] \pi, 2 \pi[, \quad u(t, x)=\sin (t \sqrt{2}) \sin x .
\end{aligned}
$$

It is obvious that the formulas (2.1.27) give essentially different values for the function $t \mapsto u_{x}(t, \pi)$ on $[0, \tau]$ and therefore contradict the regularity property established in Proposition 1.1.

Therefore we have the property

$$
\forall \tau>0, \exists t \in[0, \tau] \text { such that } u(t, \pi) \neq 0 .
$$

Now (2.1.26) and (2.1.28) obviously imply that for $x=\pi$, none of the properties (2.1.8) and (2.1.9) is satisfied (take $J=[-4 \pi, 0]$ ).

REMARK 2.1.7. The difficulty which appears in this example is not related to regularity: in fact, similar examples can be constructed with $h \in C^{\infty}(\mathbf{R} \times \bar{\Omega})$.

REMARK 2.1.8. The result of Theorem 2.1.3 is optimal for any function $\rho \in$ $L^{\infty}(\Omega)$ such that $\rho \geq 0$. Indeed, for any $\varepsilon>0$, we can choose $u_{0} \geq 0$ such that

$$
\begin{aligned}
& u_{0} \in C^{\infty}(\bar{\Omega}) \cap H_{0}^{1}(\Omega), \\
& \left.u_{0}(x)>0, \quad \forall x \in\right] l-\varepsilon, l[, \\
& u_{0}(x)=0, \quad \forall x \in[0, l-\varepsilon] .
\end{aligned}
$$


Let $u$ be the only solution of (2.1.7) such that $u_{0}(0, x)=u_{0}(x)$ and $u_{t}(0, x)=0$.

The odd extension $\tilde{u}$ of $u$ on $\mathbf{R} \times]-l, l$, as a consequence of Lemmas 1.2.2 and 1.3.2, is such that $\tilde{u} \equiv 0$ in the characteristic square with diagonal $\{0\} \times]-l+\varepsilon, l-\varepsilon[$. Hence we have for example

$$
\forall x \in] 0, \varepsilon[, \forall t \in[-l+2 \varepsilon, l-2 \varepsilon], \quad u(t, x)=0 .
$$

On the other hand, by Lemma 1.3 .2 we cannot have $u \equiv 0$ on $\mathbf{R} \times] 0, \varepsilon[$.

Finally there exists $\left.x_{0} \in\right] 0, \varepsilon\left[\right.$ such that $u\left(t, x_{0}\right)=0$ on $J$ with $|J| \geq 2(l-2 \varepsilon)$ and $u\left(t, x_{0}\right)$ is not identically zero for $t \in \mathbf{R}$.

This concludes our remark since $\varepsilon>0$ can be taken arbitrarily small. Incidentally this construction also works with $h(t, x)$ instead of $\rho(x)$ and shows that the result of Theorem 2.1.1 is also optimal for all $h(t, x)$.

2.2. The main results for semilinear wave equations in $Q$. Throughout this paragraph, we consider a numerical function $g: J \times \mathbf{R} \rightarrow \mathbf{R}$ such that

$-g$ is measurable in $t \in J$ for any $u \in \mathbf{R}$ fixed,

$-g$ is continuous in $u \in \mathbf{R}$ for almost all $t \in J$,

-if $M>0$ is given, there exists $C(M)$ such that we have for almost all $t \in J$

$$
|u| \leq M,|v| \leq M \Rightarrow|g(t, u)-g(t, v)| \leq\left. C(M)|u-v|\right|^{\bullet}
$$

In addition, we assume that $g$ satisfies the following properties:

(2.2.2) For a.e. $t \in J$, the map $u \mapsto g(t, u)$ is nondecreasing.

For almost every $t \in J$, we have

$$
\forall u \in \mathbf{R}, \quad g(t,-u)=-g(t, u) .
$$

For the following theorem, we shall consider a solution $u \in H^{1}(J \times \Omega)$ of the problem

$$
\begin{aligned}
& u_{t t}-u_{x x}+g(t, u)=0 \quad \text { in } D^{\prime}(J \times \Omega), \\
& u(t, 0)=u(t, l)=0, \quad \forall t \in J .
\end{aligned}
$$

We will denote by $\tilde{u}$ the extension of $u$ in $J \times \mathbf{R}$ which is odd and $2 l$-periodic with respect to $x$.

THEOREM 2.2.1. Assume that $|J| \geq 2 l$ and $u \not \equiv 0$ in $Q=J \times \Omega$. We set

$$
d=\inf \{x \in \Omega, u(t, x)=0, \forall t \in J\} .
$$

Then we have the following properties:

$\left(1^{\circ}\right)$ There exists $k \in \mathbf{N}, k \neq 0$ such that $d=l / k$.

$\left(2^{\circ}\right)$ For any integer $m \in\{0, \ldots, k\}$ we have

$$
\forall t \in J, \quad u(t, m l / k)=0 .
$$

$\left(3^{\circ}\right)$ Let $x_{0} \in \Omega$ be such that $\forall m \in\{0, \ldots, k\}, x_{0} \neq m l / k$. Then if $I \subset J$ is any time interval with length $\geq 2 l / k$, the function $t \mapsto u\left(t, x_{0}\right)$ must take positive and negative values on the interval $I$.

$\left(4^{\circ}\right)$ The number $2 d=2 l / k$ is the smallest period of the function $x \mapsto \tilde{u}(t, x) \in$ $L^{2}(J)$ and for any $m \in\{0, \ldots, k\}$ we have

$$
\forall(t, x) \in J \times \mathbf{R}, \quad \tilde{u}(t, 2 m l / k-x)=-\tilde{u}(t, x) .
$$


PROOF. As a consequence of (2.2.3) and Lemma 1.2.2, the function $\tilde{u}$ is a solution of

$$
\begin{aligned}
& \tilde{u}_{t t}-\tilde{u}_{x x}+g(t, \tilde{u})=0 \quad \text { in } D^{\prime}(J \times \mathbf{R}), \\
& \tilde{u}(t,-x)=\tilde{u}(t, x) \quad \text { on } J \times \mathbf{R}, \\
& \tilde{u}(t, x+2 l)=\tilde{u}(t, x) \quad \text { on } J \times \mathbf{R} .
\end{aligned}
$$

We will rely on the following

LEMMA 2.2.2. Let $x_{0} \in \Omega$ be such that $u\left(t, x_{0}\right) \geq 0$ for all $t \in J$. Then if $|J| \geq 2|\Omega|$ we have

$$
\tilde{u}\left(t, x+2 x_{0}\right)=\tilde{u}(t, x), \quad \forall(t, x) \in J \times \mathbf{R} .
$$

PROOF OF LEMMA 2.2.2. We introduce

$$
w(t, x)=\tilde{u}(t, x)+\tilde{u}\left(t, 2 x_{0}-x\right), \quad \forall(t, x) \in J \times \mathbf{R} .
$$

As a consequence of properties (2.2.1)-(2.2.3), we have

$$
g(t, \tilde{u}(t, x))+g\left(t, \tilde{u}\left(t, 2 x_{0}-x\right)\right)=h(t, x) w(t, x)
$$

where $h \in L_{\text {loc }}^{\infty}\left(J, L^{\infty}(\Omega)\right)$ and $h \geq 0$ in $Q$.

Therefore, $w$ is a solution of

$$
\begin{aligned}
& w_{x x}-w_{t t}=h(t, x) w(t, x) \quad \text { in } D^{\prime}(Q), \\
& w\left(t, x_{0}\right)=2 u\left(t, x_{0}\right), \quad \forall t \in J, \\
& w_{x}\left(t, x_{0}\right)=0, \quad \text { a.e. in } J .
\end{aligned}
$$

Since $|J| \geq 2 l$, we may assume, by performing a translation on $t$, that $]-l, l[\subset J$.

Let $\tau$ be as in $\S 1.1$ with $a=l$ and the roles of $(x, t)$ exchanged. As a consequence of Lemma 1.3.2 applied to an arbitrary "subtriangle" $\tau_{\varepsilon}$ with $l$ replaced by $l-\varepsilon$, we obtain $w(t, x) \geq 0$ in

$$
\tau=\{(t, x) \in]-l, l\left[\times \mathbf{R}, x \geq x_{0},|t|<l-\left(x-x_{0}\right)\right\} .
$$

Since $w\left(t, 2 x_{0}-x\right)=w(t, x)$ we have in fact $\left.w(t, x) \geq 0, \forall(t, x) \in\right]-l, l[\times \mathbf{R}$ such that

$$
\left|x-x_{0}\right|+|t|<l \text {. }
$$

By taking $t=0$ in (2.2.8), we find

$$
\forall x \in\left[x_{0}-l, x_{0}+l\right], \quad \tilde{u}(0, x)+\tilde{u}\left(0,2 x_{0}-x\right) \geq 0 .
$$

Since $\tilde{u}$ is odd with respect to $x,(2.2 .9)$ implies

$$
\forall x \in\left[x_{0}-l, x_{0}+l\right], \quad \tilde{u}(0,-x) \leq \tilde{u}\left(0,2 x_{0}-x\right) .
$$

By changing $x$ to $(-x)$ in this last formula, we find

$$
\forall x \in\left[-x_{0}-l,-x_{0}+l\right], \quad \tilde{u}(0, x) \leq \tilde{u}\left(0,2 x_{0}+x\right) .
$$

Since $\tilde{u}(0, x)$ is $2 l$-periodic, from (2.2.10) we deduce $\forall x \in \mathbf{R}, \quad \tilde{u}\left(0,2 x_{0}+x\right) \geq \tilde{u}(0, x)$. 
Now the function $z(x)=\tilde{u}\left(0,2 x_{0}+x\right)-\tilde{u}(0, x)$ is $\geq 0$ by $(2.2 .11)$, but also $2 l$-periodic with mean value 0 : therefore $z \equiv 0$ and we have

$$
\forall x \in \mathbf{R}, \quad \tilde{u}\left(0,2 x_{0}+x\right)=\tilde{u}(0, x) .
$$

On the other hand, from (2.2.8) we deduce that for any $h \in]-l, l[$, we have

$$
\forall x \in\left[x_{0}-l+|h|, x_{0}+l-|h|\right], \quad \tilde{u}\left(h, 2 x_{0}+x\right) \geq \tilde{u}(h, x) .
$$

By subtracting (2.2.12) from (2.2.13), dividing through by $h \neq 0$ and letting $h \rightarrow 0$, we immediately obtain that we have

$$
\left.\tilde{u}_{t}\left(0,2 x_{0}+x\right)=\tilde{u}_{t}(0, x), \quad \text { a.e. on }\right] x_{0}-l, x_{0}+l[\text {. }
$$

Since $\tilde{u}_{t}(0, y+2 l)=\tilde{u}_{t}(0, y)$ a.e. on $\mathbf{R}$, from (2.2.14) we deduce that in fact

$$
\tilde{u}_{t}\left(0,2 x_{0}+x\right)=\tilde{u}_{t}(0, x) \text { a.e. on } \mathbf{R} \text {. }
$$

From (2.2.12) and (2.2.15) we conclude that (2.2.6) is satisfied. Hence Lemma 2.2.2 is completely proved.

PROOF OF THEOREM 2.2.1 CONTINUED. We recall that for any fixed $t \in J$, the function $x \mapsto \tilde{u}(t, x)$ is periodic with period $2 l$. The set of periods of this function is, for every $t \in J$, a closed additive subgroup of $\mathbf{R}$. Now Lemma 2.2.2 and the definition of $d$ clearly imply that if either $d=0$ or $d / l \notin \mathbf{Q}$, then $\tilde{u} \equiv 0$ on $J \times \mathbf{R}$ and $u \equiv 0$ on $J \times \Omega$. Therefore if $u \not \equiv 0$ we set $d=m l / k$ with $k$ a positive integer, $m$ an integer in $[1, k]$ and $m \cap k=1$. By Bezout's Theorem, we have

$$
1 \in m \mathbf{Z}+k \mathbf{Z} \Rightarrow 2 l / k \in 2 d \mathbf{Z}+2 l \mathbf{Z} \text {. }
$$

Hence $\tilde{u}(t, x)$ is, for any $t \in J$, periodic with respect to $x$ with period $2 l / k$, and since $\tilde{u}$ is odd as a function of $x$, we deduce

$$
\forall(t, x) \in J \times \mathbf{R}, \quad \tilde{u}(t, 2 l / k-x)+\tilde{u}(t, x)=0 .
$$

By taking $x=l / k$ in (2.2.16) we find $\forall t \in J, \tilde{u}(t, l / k)=0$. Hence $d=l / k$ and $\left(1^{\circ}\right)$ is proved.

Since $\tilde{u}(t, 0)=0$ and $\tilde{u}(t, x)$ is $2 l / k$-periodic in $x,\left(2^{\circ}\right)$ is an immediate consequence of $\left(1^{\circ}\right)$.

In order to prove $\left(3^{\circ}\right)$, we consider for example $x_{0} \in \Omega, m l / k<x_{0}<(m+1) l / k$ such that $u\left(t, x_{0}\right) \geq 0$ on $I$, with $m$ an integer in $\{0, \ldots, k-1\}$ and $I$ an (open) interval with length $\geq 2 l / k$.

Since $\tilde{u}(t, x)$ is $2 l / k$-periodic in $x$, we may assume that $m=0$. Then by applying Lemma 2.2.2 with $] 0, l[$ replaced by $] 0, l / k[$ we obtain

$$
\forall(t, x) \in I \times \mathbf{R}, \quad \tilde{u}\left(t, x+2 x_{0}\right)=\tilde{u}(t, x) .
$$

This property obviously implies the stronger result

$$
\forall(t, x) \in J \times \mathbf{R}, \quad \tilde{u}\left(t, x+2 x_{0}\right)=\tilde{u}(t, x) .
$$

From (2.2.17), we deduce as in the proof of (2.2.16)

$$
\forall(t, x) \in J \times \mathbf{R}, \quad \tilde{u}\left(t, 2 x_{0}-x\right)+\tilde{u}(t, x)=0 .
$$

Finally by plugging $x=x_{0}$ in (2.2.18), we find $\forall t \in J, \tilde{u}\left(t, x_{0}\right)=0$. This gives a contradiction since $0<x_{0}<d=l / k$. 
By the same method, we show that the eventuality $u\left(t, x_{0}\right) \leq 0$ on $I$ with $I$ and $x_{0}$ as above is excluded. Hence $\left(3^{\circ}\right)$ is completely proved. The second part of $\left(4^{\circ}\right)$ is obvious. Finally the smallest period $\tau$ in question is of the form $m l / k$ and divides $2 l / k$. On the other hand if $\tau=l / k$ we find (since $\tilde{u}$ is odd with respect to $x): \tilde{u}(t, \tau / 2) \equiv 0$ on $J$ and this contradicts $\left(3^{\circ}\right)$.

REMARK 2.2.3. Let $u \not \equiv 0$ be a solution of (2.2.4) and consider the finite set $\mathcal{N}=\{0, l / k, \ldots, l\}$. Theorem 2.2.1 says that the "frequency" of the oscillations of $u(t, x)$ at any point $x_{0} \in \Omega$ with $x_{0} / l \notin \mathbf{Q}$ is, roughly speaking, increasing proportionally to the number $k=\# \mathcal{N}-1$. The case where $\mathcal{N} \neq\{0, l\}(k>1)$ is exceptional and associated to symmetry and periodicity properties with respect to $x$.

When $k>1$, it suffices to divide $\Omega$ into $k$ parts $] m l / k,(m+1) l / k[$ with $m \in$ $\{0, \ldots, k-1\}$ and one is reduced to the "generic" situation $k=1$ by looking at the solutions in $\left.\Omega^{\prime}=\right] 0, l / k[$. Indeed, we have $u(t, 0)=u(t, l / k)=0$ for $t \in J$, and the solution $u$ appears as the restriction in $\Omega$ of the extension (in the sense of Lemma 1.2.2) of $u$ considered in $\Omega^{\prime}$. Finally we notice that the results of Theorem 2.2.1 are quite significant even in the case of the ordinary wave equation. The set $\mathcal{N}$ of "nodes" gives an extension in the general case for the usual notion corresponding to harmonic oscillations of a string with fixed ends.

REMARK 2.2.4. When $g$ does not depend on $t$ and satisfies (2.2.1)-(2.2.3), any solution of (2.2.4) is in fact a global solution and the energy

$$
E=\frac{1}{2} \int_{\Omega}\left(u_{x}^{2}+u_{t}^{2}\right) d x+\int_{\Omega} G(u) d x
$$

with $G(u)=\int_{0}^{u} g(v) d v$ is conserved along the trajectories of (2.2.4). In such a case, it is natural to look at the asymptotic behavior of the solutions $u(t, x)$ as $t \rightarrow \pm \infty$. The results of Theorem 2.2.1 are helpful for that purpose. We now state

THEOREM 2.2.5. Let $g$ be as in Remark 2.2.4, and let $u$ be any solution of (2.2.4) (with $J=\mathbf{R}$ ). Then we have the following alternative:

(i) either $\lim _{t \rightarrow+\infty} \operatorname{Sup}_{x \in \Omega}|u(t, x)|=0$,

(ii) or for any $x_{0} \in \Omega$ such that $x_{0} / l \notin \mathbf{Q}$, we have

$$
\liminf _{t \rightarrow+\infty} u\left(t, x_{0}\right)<0<\limsup _{t \rightarrow+\infty} u\left(t, x_{0}\right) .
$$

ProOF. Assume that (2.2.19) is violated with for example

$$
\liminf _{t \rightarrow+\infty} u\left(t, x_{0}\right) \geq 0
$$

while $x_{0} \in \Omega$ and $x_{0} / l \notin \mathbf{Q}$.

Let $\left\{t_{n}\right\}$ be any sequence of reals with $\lim _{n \rightarrow+\infty}\left(t_{n}\right)=+\infty$. We may replace $\left\{t_{n}\right\}$ by a subsequence for which

$$
\begin{aligned}
& u\left(t_{n}, x\right) \rightarrow \varphi(x) \quad \text { in } H_{0}^{1}(\Omega) \text { weak, } \\
& u\left(t_{n}+t, x\right) \rightarrow v(t, x) \quad \text { in } L^{2}\left(a, b ; H_{0}^{1}(\Omega)\right) \text { weak }
\end{aligned}
$$

for all $(a, b) \in \mathbf{R}^{2}$ with $a<b$.

Since $u(t, x) \in L^{\infty}\left(\mathbf{R}, H_{0}^{1}(\Omega)\right) \cap W^{1, \infty}\left(\mathbf{R}, L^{2}(\Omega)\right)$, as a consequence of Lemma 1.4.1, we also have $u\left(t_{n}+t, x\right) \rightarrow v(t, x)$ in $C([a, b] \times \bar{\Omega})$ for all $(a, b)$ as above. Since $u(t, x)$ remains in a bounded subset of $\mathbf{R}$, by $(2.2 .1)$ we find

$$
g\left(u\left(t_{n}+t, x\right)\right) \rightarrow g(v(t, x)) \quad \text { in } C([a, b] \times \bar{\Omega}) .
$$


Then obviously $v$ is a solution of (2.2.4) and we must have

$$
\begin{aligned}
& \forall x \in \bar{\Omega}, \quad v(t, x)=\varphi(x), \\
& v\left(t, x_{0}\right) \geq 0, \quad \forall t \in \mathbf{R} .
\end{aligned}
$$

We claim that (2.2.21) implies $\varphi \equiv 0$.

Indeed, Theorem 2.2.1 gives $v \equiv 0$ on $\mathbf{R} \times \Omega$, and this implies the claim.

Hence for any sequence $t_{n} \rightarrow+\infty$, there exists a subsequence $\theta_{k}$ such that $u\left(\theta_{k}, x\right) \rightarrow 0$ in $L^{2}(\Omega)$ as $k \rightarrow+\infty$, and because $u(t, \cdot)$ remains in a compact subset of $C(\bar{\Omega})$, this last conclusion means that we are in case (i). The proof is similar if we assume lim $\sup _{t \rightarrow+\infty} u\left(t, x_{0}\right) \leq 0$ instead of $(2.2 .20)$.

REMARKS 2.2.6. (a) We do not know whether the case (i) can really happen with $u \neq \equiv 0$. Notice that the energy conservation is not enough to exclude a priori this possibility.

(b) On the other hand, the results of [7] (cf. also [1]) show clearly that (i) is not always satisfied, even for a strongly nonlinear function $g(u)$ of the form $c|u|^{\alpha-1} u$, $c>0, \alpha>1$.

3. Generalizations of the main results. In this section, we describe several generalizations of the main results of $\S 2$ which are obtained by similar methods.

3.1. Oscillations of $u_{x}$ at an arbitrary point. Let $\Omega$ and $J$ be as in $\S 2$ and $g: J \times \mathbf{R} \rightarrow \mathbf{R}$ a function measurable in $t$, continuous in $u$ and which satisfies (2.2.1) and (2.2.2), with $g(t, 0) \in L^{\infty}(J)$.

We consider $u \in H^{1}(J \times \Omega)$ such that

$$
u_{t t}-u_{x x}+g(t, u)=0 \text { in } D^{\prime}(J \times \Omega) .
$$

Whenever $u$ satisfies one of the boundary conditions considered in Definition 1.2.1, we shall denote by $\tilde{u}$ the extension of $u$ given by Lemma 1.2.2.

The following lemma will be essential in order to describe the oscillatory properties of $u_{x}$.

LEMMA 3.1.1. Assume that $|J| \geq l=|\Omega|$ and let $u$ be a solution of (3.1.1) with the periodic boundary conditions in $\Omega$.

If for some $x_{0} \in \bar{\Omega}$ and some interval $I \subset J$ such that $|I| \geq l$ we have either

$$
u_{x}\left(t, x_{0}\right) \geq 0 \quad \text { a.e. on } I
$$

or

$$
u_{x}\left(t, x_{0}\right) \leq 0 \quad \text { a.e. on } I \text {. }
$$

Then in fact we have

$$
\begin{gathered}
u_{x}\left(t, x_{0}\right)=0 \quad \text { a.e. on } J \\
\tilde{u}(t, x)=\tilde{u}\left(t, 2 x_{0}-x\right), \quad \forall(t, x) \in J \times \mathbf{R} .
\end{gathered}
$$

PROOF. By Lemma 1.2.2 we have

$$
\tilde{u}_{t t}-\tilde{u}_{x x}+g(t, \tilde{u})=0 \quad \text { in } D^{\prime}(J \times \mathbf{R}) .
$$

We define

$$
w(t, x)=\tilde{u}(t, x)-\tilde{u}\left(t, 2 x_{0}-x\right), \quad \forall(t, x) \in J \times \mathbf{R} .
$$


From (3.1.5) and the properties of $g$ we deduce

$$
w_{x x}-w_{t t}=h(t, x) w \quad \text { in } D^{\prime}(J \times \mathbf{R})
$$

with $h \in L^{\infty}(J \times \mathbf{R})$ and $h \geq 0$.

Also $w$ is $l$-periodic with respect to $x$ and satisfies

$$
w\left(t, x_{0}\right)=0, \quad w_{t}\left(t, x_{0}\right)=2 u_{x}\left(t, x_{0}\right) \quad \text { a.e. on } J .
$$

If for example (3.1.2) is satisfied, we may assume that $|I|=l$ and $\bar{I}=\left[t_{0}-\right.$ $\left.l / 2, t_{0}+l / 2\right]$. From Lemma 1.3.2 we deduce that

$$
w\left(t_{0}, x_{0}+\frac{l}{2}\right) \geq \int_{t_{0}-l / 2}^{t_{0}+l / 2} u_{x}\left(s, x_{0}\right) d s .
$$

On the other hand we have

$$
w\left(t_{0}, x_{0}+l / 2\right)=\tilde{u}\left(t_{0}, x_{0}+l / 2\right)-\tilde{u}\left(t_{0}, x_{0}-l / 2\right)=0 .
$$

Hence (3.1.2) and (3.1.8) imply

$$
u_{x}\left(s, x_{0}\right)=0 \quad \text { a.e. on }\left[t_{0}-l / 2, t_{0}+l / 2\right] \text {. }
$$

From (3.1.7), (3.1.9) and Lemma 1.3.2 we obtain

$$
w(t, x)=0 \text { for }\left|t-t_{0}\right|+\left|x-x_{0}\right| \leq l / 2 .
$$

From (3.1.10) it is immediate to deduce that

$$
w\left(t_{0}, x\right)=w_{t}\left(t_{0}, x\right)=0 \quad \text { a.e. on }\left[x_{0}-l / 2, x_{0}+l / 2\right] .
$$

Since $w$ is $l$-periodic in $x$ this implies

$$
w\left(t_{0}, x\right)=w_{t}\left(t_{0}, x\right)=0 \quad \text { a.e. on } \mathbf{R} .
$$

From (3.1.11) and (3.1.6) it is immediate to conclude that $w=0$ on $J \times \mathbf{R}$. Hence Lemma 3.1.1 is completely proved.

We are now able to give the results analogous to Theorem 2.2.1 concerning the oscillations of $u_{x}$. We first consider the case of periodic boundary conditions which is in a sense the general case.

THEOREM 3.1.2. Let $J, u$ be as in the statement of Lemma 3.1.1 with $u$ nonconstant on $J \times \Omega$. Let $\mathcal{V}=\left\{y \in \bar{\Omega}, u_{x}(t, y)=0\right.$ a.e. on $\left.J\right\}$.

Then the following properties are satisfied:

$\left(1^{\circ}\right)$ Either $\mathcal{V}=\varnothing$, or there exists $k \in \mathbf{N}^{*}$ and $x_{0} \in[0, l / 2 k[$ such that

$$
\mathcal{V}=\bigcup_{m=0}^{m=2 k}\left\{x_{0}+\frac{m l}{2 k}\right\} \cap[0, l] \text {. }
$$

$\left(2^{\circ}\right) \quad \forall y \in \mathcal{V}, \quad \tilde{u}(t, x)=\tilde{u}(t, 2 y-x)$ on $J \times \mathbf{R}$.

$\left(3^{\circ}\right) \forall y \notin \mathcal{V}$, for any interval $I \subset J$ such that $|I| \geq l / k(|I| \geq l$ if $\mathcal{V}=\varnothing)$ we have

$$
\begin{aligned}
& \operatorname{meas}\left\{t \in I, u_{x}(t, y)>0\right\}>0, \\
& \operatorname{meas}\left\{t \in I, u_{x}(t, y)<0\right\}>0 .
\end{aligned}
$$


PROOF. If $\mathcal{V}=\varnothing$, then $\left(2^{\circ}\right)$ is void and $\left(3^{\circ}\right)$ is a consequence of Lemma 3.1.1. Therefore from now on we assume that $\mathcal{V} \neq \varnothing$. In this case too $\left(2^{\circ}\right)$ is a consequence of Lemma 3.1.1.

Hence we only have to check $\left(1^{\circ}\right)$ and $\left(3^{\circ}\right)$. In order to do this we introduce

$$
\tilde{V}=\left\{y \in \mathbf{R}, \tilde{u}_{x}(t, y)=0 \text { a.e. on } J\right\}
$$

From the regularity properties of $\tilde{u}$ we know that $\tilde{\mathcal{V}}$ is closed. Moreover it is clear that $\mathcal{V}=\tilde{\mathcal{V}} \cap \bar{\Omega}$.

Let $x_{0}=\operatorname{Min}(\mathcal{V})$ and

$$
d=\operatorname{Min}\left\{y \in \tilde{\mathcal{V}}, y>x_{0}\right\}-x_{0} .
$$

Finally let $T=\mathbf{Z} \tau$ be the group of periods of $x \mapsto \tilde{u}(\cdot, x)$ in $L^{2}(J)$. It is clear that $\tau=m l / k$ with $m, k$ in $\mathbf{N}^{*}$ and $m \cap k=1$.

From Bezout's Theorem, we have

$$
1 \in m \mathbf{Z}+k \mathbf{Z} \Rightarrow l / k \in \tau \mathbf{Z}+l \mathbf{Z} \subset T .
$$

Hence

$$
\tau=l / k
$$

Now we remark that as a consequence of (3.1.13) and (3.1.16)

$$
\begin{aligned}
\tilde{u}\left(t, 2\left(x_{0}+l / 2 k\right)-x\right) & =\tilde{u}\left(t, 2 x_{0}-(x-l / k)\right) \\
& =\tilde{u}(t, x-l / k)=\tilde{u}(t, x), \quad \forall(t, x) \in J \times \mathbf{R} .
\end{aligned}
$$

Hence

$$
\tilde{u}_{x}\left(t, x_{0}+l / 2 k\right)=0 \text { a.e. on } J .
$$

Now (3.1.17) and (3.1.15) imply $d \leq l / 2 k$.

On the other hand, for any $(t, x) \in J \times \mathbf{R}$ :

$$
\begin{aligned}
\tilde{u}(t, x) & =\tilde{u}\left(t, 2 x_{0}-x\right)=\tilde{u}\left(t, 2 x_{0}+2 d-\left(2 x_{0}-x\right)\right) \\
& =\tilde{u}(t, 2 d+x) \\
& \Rightarrow 2 d \in T .
\end{aligned}
$$

Therefore $d=l / 2 k$.

As an immediate consequence, we have $l / k \in T$, and $\bigcup_{m \in Z}\left\{x_{0}+m l / 2 k\right\} \subset \tilde{V}$.

The reverse inclusion: $\tilde{\mathcal{V}} \subset \bigcup_{m \in Z}\left\{x_{0}+m l / 2 k\right\}$ is an obvious consequence of (3.1.15) since $\tilde{u}$ is $l / k$-periodic and even in $x$ with respect to $x_{0}+d=x_{1}$.

Hence the proof of $\left(1^{\circ}\right)$ is achieved.

Finally, $\left(3^{\circ}\right)$ is an obvious consequence of $\left(1^{\circ}\right)$ and Lemma 3.1.1.

REMARK 3.1.3. It follows from (3.1.12) that

-whenever $\mathcal{V} \neq \varnothing$, it contains at least two points,

-if $x_{0}=0, \mathcal{V}$ contains $2 k+1$ points,

-if $x_{0}>0, v$ contains $2 k$ points.

These conclusions are easy to interpret in the case of elementary harmonics $\cos t \cos x$ considered on $] \alpha, \alpha+2 n \pi[$.

In the case of Neumann boundary conditions, we have the following result. 
THEOREM 3.1.4. Assume that $|J| \geq 2 l=2|\Omega|$ and let $u$ be a solution of (3.1.1) with the Neumann boundary conditions in $\Omega, u$ being nonconstant on $J \times \Omega$. Let $\mathcal{V}$ be as in the statement of Theorem 3.1.2. Then

$\left(1^{\circ}\right) \exists k \in \mathbf{N}^{*}, \mathcal{V}=\bigcup_{m=0}^{k}\{m l / k\}$,

$\left(2^{\circ}\right) \forall k \in \mathcal{V}, \tilde{u}(t, x)=\tilde{u}(t, 2 y-x)$ on $J \times \mathbf{R}$,

$\left(3^{\circ}\right) \forall y \notin \mathcal{V}$, for any interval $I \subset J$ such that $|I| \geq 2 l / k$, we have (3.1.14).

PROOF. $\tilde{u}$ satisfies the periodic boundary conditions on $] 0,2 l[$. Then Theorem 3.1.4 follows from Theorem 3.1.2 since $0 \in \mathcal{V}$.

Finally, we consider the case of Dirichlet boundary conditions.

THEOREM 3.1.5. Assume that $g, u$ and $J$ are as in the statement of Theorem 2.2.1. Let

$$
\begin{aligned}
& \mathcal{N}=\{y \in \bar{\Omega}, u(t, y)=0 \text { on } J\}, \\
& \mathcal{V}=\left\{y \in \bar{\Omega}, u_{x}(t, y)=0 \text { a.e. on } J\right\} .
\end{aligned}
$$

Let $k \in \mathbf{N}^{*}$ be such that $\mathcal{N}=\bigcup_{m=0}^{k}\{m l / k\}$.

Then we have the following conclusions:

$\left(1^{\circ}\right)$ Either $\mathcal{V}=\varnothing$ or $\mathcal{V}=\bigcup_{m=0}^{k-1}\{l / 2 k+m l / k\}$.

$\left(2^{\circ}\right) \forall y \in \mathcal{V}, \tilde{u}(t, x)=\tilde{u}(t, 2 y-x)$ on $J \times \mathbf{R}$.

$\left(3^{\circ}\right) \forall y \notin \mathcal{V}$, for any interval $I \subset J$ such that $|I| \geq 2 l / k$, we have (3.1.14).

PROOF. Since $g$ is assumed to be odd with respect to $x$, the extension $\tilde{u}$ is a solution on $J \times \mathbf{R}$ of $\tilde{u}_{t t}-\tilde{u}_{x x}+g(t, \tilde{u})=0$ which satisfies the periodic boundary conditions in $] 0,2 l[$.

Hence $\left(2^{\circ}\right)$ and $\left(3^{\circ}\right)$ follow from Theorem 3.1.2 since $\tilde{u}$ is not constant on $J \times] 0,2 l[$.

Assume $\mathcal{V} \neq \varnothing$. From Theorem 3.1.2 we know that $\mathcal{V}=\bigcup_{m=0}^{k}\left\{x_{0}+m l / k\right\} \cap[0, l]$ (because $2 l / k$ is the smallest period of $\tilde{u}$ with respect to $x$ ) with $x_{0} \in[0, l / k[$.

Since $\tilde{u}(t, x)=\tilde{u}\left(t, 2 x_{0}-x\right)$ on $J \times \mathbf{R}$, by taking $x=0$ we find

$$
\tilde{u}\left(t, 2 x_{0}\right)=0 \quad \text { on } J \Rightarrow x_{0} \in\{0, l / 2 k\} .
$$

If $x_{0}=0$ we conclude that $\tilde{u} \equiv 0$ on $Q$ [since then $\left.\tilde{u}(t, x)=\tilde{u}(t,-x)\right]$. Hence we must have $x_{0}=l / 2 k$ and

$$
\mathcal{V}=\bigcup_{m=0}^{k-1}\left\{\frac{l}{2 k}+\frac{m l}{k}\right\}
$$

The proof of Theorem 3.1.5 is complete.

3.2. Oscillations of $u$ in the case of Neumann or periodic boundary conditions. The results in this section will appear weaker than those of $\S \S 2.2$ and 3.1. The reason for this is rather simple: in the case of (for example) periodic boundary conditions, the kernel of $u_{t t}-u_{x x}$ contains all constant functions. When we study the oscillations of $u_{x}$, this kernel disappears: on the other hand if we want $u(t, x)$ to oscillate around 0 rather than other possible values, then we need to assume some "coerciveness" of the perturbation $g(t, u)$. Our basic tool in this paragraph will be the following. 
Lemma 3.2.1. Let $\Omega=] 0, l[$ and $a \in \mathbf{R} \cup\{-\infty\}, J=] a,+\infty[$. Assume that $g$ satisfies the hypotheses made in the statement of Theorem 2.2.1, and that in addition we have for some $p \geq 1$

$$
\forall t \in J, \forall(u, v) \in \mathbf{R}^{2}, \quad|g(t, u)-g(t, v)| \geq f(t)|u-v|^{p}
$$

where $f \in L_{\text {loc }}^{1}(J), f \geq 0$ and, with

$$
F(t)=\int_{t}^{+\infty} f(s) d s
$$

one of the following conditions is satisfied:

(3.2.2) Either $F(t)=+\infty, \forall t \in J$ or $F(t)<+\infty, \forall t \in J$, and in case $p=1$, we assume

$$
\limsup _{t \rightarrow+\infty}(t F(t))>1 .
$$

In case $p>1$, we only require

$$
\exists t_{0} \in J, \quad \int_{t_{0}}^{+\infty} F(t) d t=+\infty .
$$

Let $u$ be a solution of (3.1.1) with periodic boundary conditions in $\Omega$. Then if we assume

$$
\exists t_{1} \in J, \forall t>t_{1}, \quad u\left(t, x_{0}\right) \geq 0
$$

for some $x_{0} \in \bar{\Omega}$, we can conclude that

$$
\forall t \in J, \forall y \in \mathbf{R}, \quad \tilde{u}\left(t, 2 x_{0}-y\right)=-\tilde{u}(t, y)
$$

and as a consequence we have

$$
\begin{gathered}
\forall t \in J, \quad u\left(t, x_{0}\right)=0, \\
\forall t \in J, \forall m \in \mathbf{Z}, \quad \tilde{u}\left(t, x_{0}+m l\right)=0 .
\end{gathered}
$$

PROOF. It is clear that we can assume $J=] 0,+\infty\left[\right.$ with $a=t_{1}=0$ and $t_{0}=0$ when it is defined. We consider $w(t, x)=\tilde{u}(t, x)+\tilde{u}\left(t, 2 x_{0}-x\right)$ in $\Delta=\{(t, x) \in$ $\left.J \times \mathbf{R},\left|x-x_{0}\right|<t\right\}$.

Since $w\left(t, x_{0}\right)=2 u\left(t, x_{0}\right)$ and $w_{x}\left(t, x_{0}\right)=0$ for $t \in J$, we have by the same method as in the proof of Theorem 2.2.1:

$$
w \geq 0 \text { a.e. on } \Delta \Rightarrow w \geq 0 \text { on }] l,+\infty[\times \mathbf{R} \text { by periodicity. }
$$

Again, by translating the origin of time, we may assume that $w \geq 0$ on $J \times \mathbf{R}$. Then by using (3.2.1) and the other properties of $g$, we obtain after integration over $\Omega$ (notice that $w$ is $l$-periodic in $x$ )

By using Hölder's inequality, we obtain

$$
\frac{d^{2}}{d t^{2}} \int_{\Omega} w(t, x) d x \leq-f(t) \int_{\Omega} w^{p}(t, x) d x .
$$

$$
v^{\prime \prime} \leq-f(t) v^{p} /|\Omega|^{p-1}
$$

where

$$
v(t)=\int_{\Omega} w(t, x) d x
$$

and $v(t) \in W_{\text {loc }}^{2,1}(J)$ as is shown by a technical but easy argument.

Now the proof of Lemma 3.2.1 is reduced to the following result on nonautonomous ordinary differential equations. 
LEMMA 3.2.2. Let $v \in W_{\text {loc }}^{2,1}(J)$ be such that $v \geq 0$ on $J$ and $v$ satisfies (3.2.9), with $f(t)$ as in the statement of Lemma 3.2.1. Then in fact $v \equiv 0$ on $J$.

PROOF OF LEMMA 3.2.2. The function $v(t)$ is $\geq 0$, concave and nondecreasing on $J$.

-If for some $t_{0} \in J$ we have $v\left(t_{0}\right)=0$, then $v \equiv 0$ on $J$.

-If on the contrary $v>0$ on $J$, let

$$
\varphi(t)=v^{\prime} / v^{p}, \quad \forall t \in J .
$$

From (3.2.9) we deduce immediately

$$
\varphi^{\prime}(t)+p v^{\prime 2} / v^{p+1} \leq-f_{1}(t), \quad \text { a.e. on } J
$$

with $f_{1}(t)=f(t) /|\Omega|^{p-1}$.

It is clear from (3.2.12) that $\varphi(t)$ is nonincreasing. Since $\varphi(t) \geq 0$ on $J$, by integrating (3.2.12) on $] 0,+\infty[$ we deduce

$$
\int_{t}^{+\infty} f_{1}(s) d s \leq \varphi(t), \quad \forall t \in J .
$$

As a consequence, Lemma 3.2.2 is already proved if $f(t)$ satisfies (3.2.2).

Now we consider the case $p=1$. Then (3.2.12) implies in particular

$$
\varphi^{\prime}(t)+\varphi^{2}(t) \leq 0 \Rightarrow \varphi(t)<1 / t, \quad \forall t \in J .
$$

By plugging this inequality in (3.2.13), we obtain

$$
F(t)=\int_{t}^{+\infty} f(s) d s=\int_{t}^{+\infty} f_{1}(s)<\frac{1}{t}, \quad \forall t \in J .
$$

It is obvious that (3.2.14) contradicts (3.2.3). Hence Lemma 3.2 .2 is proved in this case.

Finally we assume that $p>1$. Then $(3.2 .13)$ can be rewritten in the form

$$
F(t) \leq|\Omega|^{p-1} \varphi(t)=|\Omega|^{p-1} \frac{d}{d t}\left(-\frac{1}{(p-1)[v(t)]^{p-1}}\right) .
$$

Since $v(t)>0$ on $J$ and $v$ is nondecreasing, it is clear that $1 / v(t)$ is bounded on $\left[t_{0},+\infty\left[\right.\right.$ for any $t_{0} \in J$. Hence (3.2.15) implies

$$
\int_{t_{0}}^{+\infty} F(t) d t \leq \frac{|\Omega|^{p-1}}{(p-1)\left[v\left(t_{0}\right)\right]^{p-1}}<+\infty .
$$

It is clear that (3.2.16) contradicts (3.2.4). Therefore the proof of Lemma 3.2.2 is completed.

END OF PROOF OF LEMMA 3.2.1. From Lemma 3.2.2 we deduce that $v(t)=$ $\int_{\Omega} w(t, x) d x$ is identically zero for $t \in J$. In fact, we obtain that $w(t, x) \equiv 0$ on ]$t_{2},+\infty[\times \Omega$, and it follows easily (as already remarked previously) that (3.2.6) is satisfied. Then (3.2.7) and (3.2.8) are obvious and the proof of Lemma 3.2.1 is completed. 
THEOREM 3.2.3. Let $\Omega, J$ and $g$ be as in the statement of Lemma 3.2.1 and let $u \not \equiv 0$ be a solution of (3.1.1) with the periodic boundary conditions in $\Omega$. Let $\mathcal{N}=\{y \in \bar{\Omega}, u(t, y)=0$ on $J\}$.

Then we have two possible cases.

$\left(1^{\circ}\right)$ If $\mathcal{N}=\varnothing$, then for any $x \in \bar{\Omega}$ the function $t \mapsto u(t, x)$ takes positive and negative values on any interval of the form $] \tau,+\infty[\subset J$.

$\left(2^{\circ}\right)$ If $\mathcal{N} \neq \varnothing$, there is $k \in \mathbf{N}^{*}$ and $x_{0} \in[0, l / 2 k[$ such that

$$
\mathcal{N}=\bigcup_{m=0}^{2 k}\left\{x_{0}+m l / 2 k\right\} \cap \bar{\Omega} \text {. }
$$

\section{Furthermore}

(i) If $y \in \mathcal{N}$, then $\tilde{u}(t, x)=-\tilde{u}(t, 2 y-x)$ on $J \times \mathbf{R}$.

(ii) If $y \in \Omega \backslash \mathcal{N}$, then the function $t \mapsto u(t, y)$ takes positive and negative values on any subinterval $I \subset J$ such that $|I| \geq l / k$.

Proof. $\left(1^{\circ}\right)$ is an obvious consequence of Lemma 3.2.1. Assume now $\mathcal{N} \neq \varnothing$ and let $x_{0}=\operatorname{Min} \mathcal{N}$. We set $\varphi(t, x)=\tilde{u}\left(t, x_{0}+x\right), \forall(t, x) \in J \times \mathbf{R}$. Clearly $\varphi$ is a solution of (3.1.1) which is $l$-periodic with respect to $x$ and satisfies Dirichlet boundary conditions in $\Omega$.

Let $\mathcal{M}=\{x \in \bar{\Omega}, \varphi(t, x)=0$ on $J\}$. By Theorem 2.2.1, we know that there exists $k^{\prime} \in \mathrm{N}^{*}$ such that $\mathcal{M}=\bigcup_{m=0}^{k^{\prime}}\left\{m l / k^{\prime}\right\}$. In addition $2 l / k^{\prime}$ is the minimal period of $\varphi$ with respect to $x$. Since $l$ is also a period of $\varphi$, there exists $k \in \mathbf{N}^{*}$ such that $l=2 l k / k^{\prime} \Rightarrow k^{\prime}=2 k$.

On the other hand, since $\tilde{u}\left(t, x_{0}-l / 2 k\right)=0$ on $J$ we have $x_{0} \in[0, l / 2 k[$. Hence the first assertion of $\left(2^{\circ}\right)$ is satisfied since $\mathcal{N}=\left(x_{0}+\mathcal{M}\right) \cap \bar{\Omega}$.

Finally (i) and (ii) are obvious consequences of Theorem 2.2.1 applied to $\varphi$.

THEOREM 3.2.4. Let $\Omega, J$ and $g$ be as in the statement of Lemma 3.2.1 and let $u \not \equiv 0$ be a solution of (3.1.1) with Neumann boundary conditions in $\Omega$. Let $\mathcal{N}=\{y \in \bar{\Omega}, u(t, y)=0$ on $J\}$.

Then we have two possible cases.

$\left(1^{\circ}\right)$ If $\mathcal{N}=\varnothing$, then for any $x \in \bar{\Omega}$ the function $t \mapsto u(t, x)$ takes positive and regative values on any interval of the form $] \tau,+\infty[\subset J$.

$\left(2^{\circ}\right)$ If $\mathcal{N} \neq \varnothing$, there is $k \in \mathbf{N}^{*}$ such that $\mathcal{N}=\bigcup_{m=0}^{k-1}\{l / 2 k+m l / k\}$. Furthermore,

(i) If $y \in \mathcal{N}$, then $\tilde{u}(t, x)=-\tilde{u}(t, 2 y-x)$ on $J \times \mathbf{R}$.

(ii) If $y \in \bar{\Omega} \backslash \mathcal{N}$, then the function $t \mapsto u(t, y)$ takes positive and negative values on each interval $I \subset J$ with $|J| \geq 2 l / k$.

PROOF. $\tilde{u}$ is a solution of (3.1.1) which satisfies the periodic boundary conditions on $] 0,2 l[$.

On applying Theorem 3.2 .3 we obtain $\left(1^{\circ}\right),\left(2^{\circ}\right)$ (i) and $\left(2^{\circ}\right)$ (ii). In addition, there exists $x_{0} \in[0, l / k[$ such that

$$
\mathcal{N}=\bigcup_{m=0}^{k}\left\{\frac{m l}{k}+x_{0}\right\} \cap \bar{\Omega}, \quad k \in \mathbf{N}^{*} .
$$

If $x_{0}=0$, then clearly $\tilde{u} \equiv 0$ and $u \equiv 0$ which is excluded by hypothesis.

On the other hand we have $0=\tilde{u}\left(t, x_{0}\right)=\tilde{u}\left(t,-x_{0}\right)$ on $J$. It follows that $2 x_{0}=l / k \Rightarrow x_{0}=l / 2 k$. 
REMARK 3.2.5. The hypotheses of Theorem 3.2.3 and Lemma 3.2.1 are rather sharp. Indeed, the function $u(t, x)=\sqrt{t}$ is a solution of $u^{\prime \prime}=-\frac{1}{4} u(t) / t^{2}$ on $J=] 0,+\infty[$.

Hence it is a particular solution of (3.1.1) with periodic boundary conditions in $\Omega$ and with $g(t, u)=u / 4 t^{2}$. Inequality (3.2.1) is here satisfied with $p=1$ and $f(t)=1 / 4 t^{2} \Rightarrow F(t)=1 / 4 t$. Hence Theorem 3.2.3 can become false if (3.2.3) is replaced by $\liminf \operatorname{int}_{t \rightarrow+\infty} t F(t)>0$.

Also, let $u_{\varepsilon}(t, x)=t^{\varepsilon}$ for $t>0$. Then

$$
u_{\varepsilon}^{\prime \prime}=-\varepsilon(1-\varepsilon) t^{\varepsilon-2}, \quad u_{\varepsilon}^{p}=t^{p \varepsilon} .
$$

Hence

$$
u_{\varepsilon}^{\prime \prime} / u_{\varepsilon}^{p}=-\varepsilon(1-\varepsilon) t^{\varepsilon} / t^{2+p \varepsilon},
$$

and for any $p>1$ fixed, $u_{\varepsilon}$ is a positive solution of (3.1.1) with periodic boundary conditions and

$$
f(t)=\varepsilon(1-\varepsilon) / t^{2+p \varepsilon-\varepsilon} \Rightarrow F(t)=C(\varepsilon) / t^{1+(p-1) \varepsilon} .
$$

Hence $F(t)$ can be like a power of $t^{-1}$ arbitrary close to $t^{-1}$ which satisfies (3.2.4).

Most probably these hypotheses can still be refined but this is not our main point here.

3.3. Concluding remarks. In this paper, we have focused our attention on the problem of the "oscillations around zero" of the function $u(t, x)$ where $\Omega=] 0, l[$, $x \in \Omega$ is fixed and $u$ is the general solution of a semilinear wave equation of the form (3.1.1) satisfying homogeneous boundary conditions at the endpoints 0 and $l$.

It is clear that the oscillation properties are strongly related to boundary conditions. On the other hand, the one-dimensional character and the hypothesis (made in several statements) that $g(t,-u)=-g(t, u)$ do not seem at first sight to be so necessary.

In fact, part of the methods that we used in this paper can exploited in other situations:

- If we consider the equation $\square u+g(t, u)=0$ in a ball of $\mathbf{R}^{n}$ with $n>1$, a method rather similar to the proof of Theorem 2.1.1 provides oscillation results for radially symmetric solutions with Dirichlet boundary conditions, at any point $x_{0}$ close to the boundary (cf. [3]).

-If $\Omega=] 0, l\left[\right.$ and $g(t, u) \equiv u^{+}$, the condition $u(t, l / 2) \geq 0$ on $J$ with $|J|>l$ implies that $u \equiv 0$ on $\mathbf{R} \times \Omega$. However we do not know what happens concerning the points $x_{0}$ such that $x_{0} / l \notin \mathbf{Q}$ for example. An important remark, showing that the method of proof of Theorem 2.2.1 cannot be used in this case, is that there exists no extension $\tilde{u}$ of a nontrivial solution $u$ of $\square u+u^{+}=0$ which satisfies Dirichlet boundary conditions at points $0, l$ and $2 l$ for $t \in J,|J|>2 l$.

-If $\Omega=] 0, l\left[\right.$ and $g(t, u)$ is $C^{1}$ and odd with respect to $u$, the method of proof of Theorem 2.2.1 shows that if $u \neq \equiv$ is a solution of (2.2.4) on $\mathbf{R} \times \Omega$ and $x_{0} \in \Omega$ is such that $x_{0} / l \notin \mathbf{Q}$, the function $t \mapsto u\left(t, x_{0}\right)$ cannot vanish on an interval $J$ such that $|J| \geq 2 l$.

Here the monotonicity of $g$ in $u$ is not needed. This property is related to a "controllability" property of equation (2.2.4). Analogous questions are discussed by J. L. Lions in [6]. 


\section{BIBLIOGRAPHY}

1. H. Brezis, J. M. Coron and L. Nirenberg, Free vibrations for a nonlinear wave equation and a theorem of P. Rabinowitz, Comm. Pure Appl. Math. 33 (1980), 667-689.

2. T. Cazenave and A. Haraux, Propriétés oscillatoires des solutions de certaines équations des ondes semi-linéaires, C.R. Acad. Sci. Paris Sér. I Math. 298 (1983), 449-452.

3. __ Some oscillatory properties of the wave equation in several dimensions, J. Funct. Anal. (submitted).

4. R. Courant and D. Hilbert, Methods of mathematical physics, vol. II, Interscience, New York and London, 1962.

5. A. Haraux, Nonlinear evolution equation: Global behavior of solutions, Lecture Notes in Math., vol. 841, Springer-Verlag, Berlin, 1981.

6. J. L. Lions, Séminaire Goulaouic-Schwartz, Feb. 1984.

7. P. Rabinowitz, Free vibrations for a semi-linear wave equation, Comm. Pure Appl. Math. 31 (1978), 31-68.

8. I. Segal, Nonlinear semi-groups, Ann. of Math. (2) 78 (1963), 339-363.

UNiversité Pierre et MARIE CURIE, laboratoire d'ANAlyse NumériQUe (L.A. 189), Tour 55-65-5ème Étage, 4, Place Jussieu, 75252 Paris Cedex 05, France 